\title{
Lungs and hypoxia: a review of the literature
}

\author{
Mustafa F. Sargon iD \\ Department of Anatomy, Faculty of Medicine, Lokman Hekim University, Ankara, Turkey
}

\begin{abstract}
Acute, intermittent or chronic hypoxia have negative effects on lung maturation during the embryological period which has been shown by many experimental models designed on animal studies. The receptors responsible from the development of lungs in fetal period are affected from hypoxia. Hypoxia also affects the morphometry, anatomy and microscopy of lung tissue in the adults. In acute phase of hypoxia; lung parenchyma showed destructive oxidative changes. However, in later phases repair and proliferative processes were observed in the lung tissue. Damage to the lining layer of alveoli, accumulation of alveolar macrophages, oedematous changes in the lung parenchyma, mild oedema, inflammatory cell infiltration, increased number of type II pneumocytes and pulmonary fibrosis are the main findings in cases of hypoxia. Chronic hypoxia accentuates lung growth by increasing the lung parenchyma. Decrease of capillary volume and suppression of elastin repair in lung fibroblasts are other clinically important microscopic findings in hypoxia. Many molecular studies found in the literature revealed micro-RNAs to be involved in modulation of hypoxia-induced pulmonary hypertension. In animal models submitted to acute hypobaric hypoxia; the researchers detected an increase in eNOS mRNA which is responsible of the immediate response, producing nitric oxide that caused vasodilation and bronchodilation in lung tissue. In other molecular studies; suppression of many immune molecules, major changes at the levels of various enzymes and growth factors were detected in the researches. Additionally; hypoxia causes to an increase in the amount of lung cancer cells and therefore; induces the metastases of lung cancer cells to brain tissues.
\end{abstract}

Keywords: cancer; gross anatomy; hypoxia; lung; microscopy; molecular study

Anatomy 2021;15(1):76-83 @2021 Turkish Society of Anatomy and Clinical Anatomy (TSACA)

\section{Introduction}

Hypoxia describes the inability while using oxygen or it is the case of oxygen deficiency in tissues. Hypoxia itself is not a respiratory disease, but is often a consequence of respiratory diseases. According to its cause; hypoxia is classified in four groups; hypoxemic hypoxia, ischemic hypoxia, anemic hypoxia and histotoxic hypoxia. In hypoxemic hypoxia; the arterial $\mathrm{PO}_{2}$ is low and it is due to inadequate pulmonary gas exchange in most of the cases. The major causes of hypoxemic hypoxia are respiratory arrest, degenerative lung diseases, atmospheric deficiency of oxygen at high elevations and impaired ventilation. Inadequate circulation of the blood is the cause of ischemic hypoxia. Congestive heart failure is a good example to this condition. Anemic hypoxia occurs in cases of anemia and in this situation; the blood cannot carry adequate amounts of oxygen for the body. If a metabolic poison prevents the usage of oxygen by a tissue; histotoxic hypoxia occurs in these cases. ${ }^{[1]}$
Approximately 480 million alveoli are found in the human lungs. $64 \%$ of total lung structure is formed by these alveoli. Alveoli provide a surface area of 120-150 $\mathrm{m}^{2}$ dedicated for gas exchange. The oxygen diffusion constant and gas exchange surface area are proportional to body weight and oxygen consumption. Exposure to a cold environment, living in high altitudes and physical hyperactivity causes to an increase in oxygen diffusion capacity in proportion to enhanced oxygen consumption. Life-threatening barrier to proper oxygenation occurs in cases of decreased ventilatory drive, airway obstruction, intraalveolar exudate, damages to alveolar capillaries, septal thickening due to edema, inflammation and fibrosis. The alveolar/arterial $\mathrm{PO}_{2}$ gradient is enhanced if there is a life-threatining barrier to proper oxygenation. ${ }^{[2]}$

The aim of this review article is to define the embryological, gross anatomic, histopathological and molecular changes observed in the lung tissue in cases of hypoxia. Additionally; the relation of hypoxia and tumors is also discussed in the study. The manuscripts related to 
hypoxia were searched from the literature, from PubMed (https://www.ncbi.nlm.nih.gov/pubmed) in between the years of 1945-2019. From these manuscripts; the ones, which were related to lung tissue changes due to hypoxia, were chosen and included to the review. The key words selected for searching the database were "hypoxia and lungs". In the research of these keywords; totally 15,445 manuscripts were found. From these manuscripts; 43 of them were related with our subject and these studies were included to the review study.

\section{Hypoxia and Development of the Lungs}

Schwartz et al. ${ }^{[3]}$ examined fetal growth in rats exposed to brief and intermittent hypoxia with little change in nutrient intake. In rats exposed to hypoxia for one hour; fetal body weight, length of the fetus and liver weight was decreased. However; the brain/liver weight ratio was increased in these rats, when compared with the control group. In the study; another group of rats were exposed to hypoxia for two hours and results were the decrease of the fetal body weight and fetus length. The lung develops in relative hypoxic conditions if the fetus is exposed to $25 \%$ of ambient oxygen levels in utero. ${ }^{[4]}$ Hypoxic conditions in fetal environment stimulates the developmental stages of embryo, organogenesis and vascularization of the organs. ${ }^{[5,6]}$ Vascular endothelial growth factor (VEGF) is an important factor in lung development in the fetus. Inhibition of VEGF receptor signaling impairs the branching of airways and blood vessels in lung explants. Regulation of hypoxia-inducible genes and expression levels of hypoxia-inducible factors such as VEGF are necessary for proper lung development. ${ }^{[7]}$

The differential effects of hypoxic levels to organ system development of the chicken was studied by Zhang and Burggren ${ }^{[8]}$ Hypoxia occurring from day zero to day 10 during incubation had larger effects on embryonic mortality and organ growth than hypoxia occurring from day 10 to day 18. Growth of the heart and chorioallantoic membrane was stimulated by chronic hypoxia in the study. However; the lung, brain, eye, liver, stomach, beak, and toes were most affected from hypoxia. Sustained hypoxia from the beginning of incubation in chicken decreased blood haemoglobin and haematocrit levels. Red blood cell concentration of embryos at day 10 were also affected. At day 18; there was no statistically significant difference in between hypoxic and normoxic groups. In conclusion; modest hypoxia which describes $15 \%$ oxygen level during development, or hypoxia at any level during the late stages of development (from day 11 to day 18) increased the weights of the heart and chorioallantoic membrane. The results of this study showed that the first half of embryonic development contained critical parameters for the detrimental effects of hypoxia and the second half of embryonic development contained critical parameters for the compensatory responses of hypoxia in key organs.

Schmiedl et al. ${ }^{[9]}$ investigated the influence of prenatal hypoxia and/or postnatal hyperoxia on morphological lung maturation based on stereological parameters in mice. The aim of this study was to find out which model best reflects morphological changes in lung development comparable with alterations observed in bronchopulmonary dysplasia. The authors found that prenatal hypoxia and postnatal hyperoxia differentially influenced the maturation of parenchyma of lungs. After hypoxia and hyperoxia in 14-day old mice, they observed a significant retardation in lung development morphologically which lead to bronchopulmonary dysplasia like alterations.

\section{Hypoxia and Anatomic Studies}

Davies et al. ${ }^{[10]}$ developed a method based on planimetry of elastic laminae in vessel cross sections and determined the reactivity of microvessels of lung tissue. The authors obtained slices from the lungs of six rats exposed to hypobaric hypoxia for 14 days. According to their results; after chronic hypoxia, the regression line shifted which indicated the structural remodeling. After hypoxia, newly muscularized arteries were found and in these vessels; the new internal elastic lamina was always shorter than the external elastic lamina. A complex network of elastin was connecting the two laminae. Sekhon and Thurlback ${ }^{[1]]}$ studied the morphometry of lungs in rats between four and seven weeks of age. They divided the rats into five groups; control, hypobaric normoxic, normobaric hypoxic, hypobaric hypoxic, and weight matched to the hypobaric hypoxic group. In hypobaric hypoxic and normobaric hypoxic rats; the volume of the lungs, alveolar surface area of lungs and total number of alveoli were increased when compared to the weightmatched control group. In normobaric hypoxia group of rats; mean linear intercept, mean chord length of alveoli were increased and the number of alveoli per unit volume were decreased when compared to the weightmatched control animals. In hypobaric hypoxia group; the only change was the increase of mean chord length in rats. In hypobaric normoxia group; alveolar size and lung volume were diminished when compared with the control groups. Lung growth was impaired in weightmatched controls without affecting the dimension of airspaces. In hypobaric hypoxic and normobaric hypoxic rats; an increase was observed in lung growth which 
overcame the nutritional effects. In the rats; Sulkowska ${ }^{[12]}$ evaluated the effects of hypobaric hypoxia on lung parenchyma and in the surfactant system-forming structures in the lungs. These changes were evaluated basing on ultrastructural analysis by transmission electron microscopy. In the early phase; in three days, destructive-exudative changes predominated. These ultrastructural changes were delamelation of type II pneumocytes, oedematous changes in the lungs (Figure 1), damage to the lining layer of alveoli and accumulation of alveolar macrophages in the lung tissue. In the later phases, repair-proliferative processes were found to be predominant such as increased number of type II pneumocytes and focal intensification of fibroplasia (Figure 2). In hypobaric conditions for 3 and 10 days; the changes within the surfactant system-forming structures were accompanied by the accumulation of granulocytes and monocytes. In hypobaric conditions for 30 days; an accumulation of blood platelets was detected in the vascular bed of the lungs. Clough et al. ${ }^{[13]}$ used $\chi$-ray microfocal angiographic images of isolated perfused dog lung lobes in order to detect the effects of alveolar hypoxia on pulmonary microvascular volume. Their results showed that the capillary volume was decreased by hypoxia in the dogs' lung lobes. In a scanning electron microscopic study; Gade et al. ${ }^{[14]}$ examined the effects of bronchial arterial devascularization on the morphological appearance of bronchi in pigs. Bronchial arterial devascularization and bronchial transection were performed in pigs and the ultrastructure of the surfaces of bronchi was

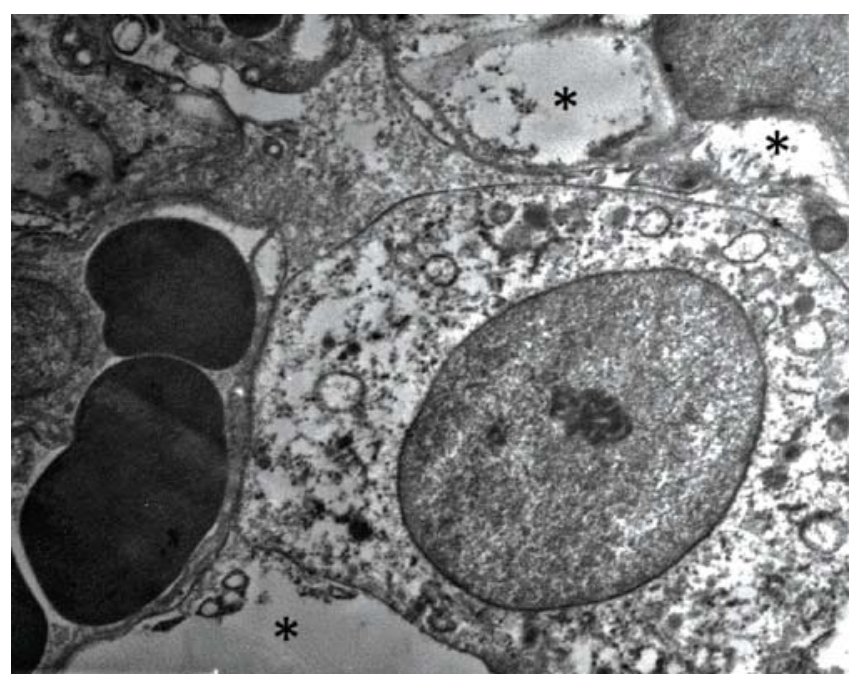

Figure 1. Transmission electron micrograph showing oedematous changes in lung tissue ${ }^{*}$ ) in hypoxia. (Original magnification $\times 5000$ ) (from the collection of author). examined. In conclusion; the grooves in between the bronchi were wrinkled in appearance and a large proportion of the epithelial cells were devoid of cilia. New cilia seemed to shoot up from the surface, in the study. On the control side; the ultrastructural morphology was similar with the normal pigs. In another study; Gade et al. ${ }^{[15]}$ examined the physiological consequences of porcine bronchial transsection and reanastomosis with and without bronchial artery blood supply with relation to lung transplantation. In the study; postoperatively, bronchial transsection and reanastomosis without bronchial artery blood supply was resulted by a significant decrease in the mucosal blood flow and saturation. Additionally; a significant decrease was detected in the tissue haemoglobin concentration. These findings provided a physiological explanation of histological changes observed in the study. In the microscopic examination of main bronchi and lung parenchyma; oedema (Figure 1), slight fibrosis (Figure 2) and inflammation were detected. In this study; the most remarkable two findings were the oedematous lung septa with inflammation and beginning of the fibrosis. In an experimental study of chronic hypoxia by Berk et al.; ${ }^{[16]}$ it was shown that the chronic hypoxia supressed elastin repair by rat lung fibroblasts. According to their data; it was suggested that regional hypoxia limited lung elastin repair following protease injury at least in part by inhibiting elastin gene expression. In cases of acute hypoxia; Miserocchi ${ }^{[17]}$ examined the lung interstitial pressure and structure of the lungs. In the study; fragmentation of chondroitin sulphate pro-

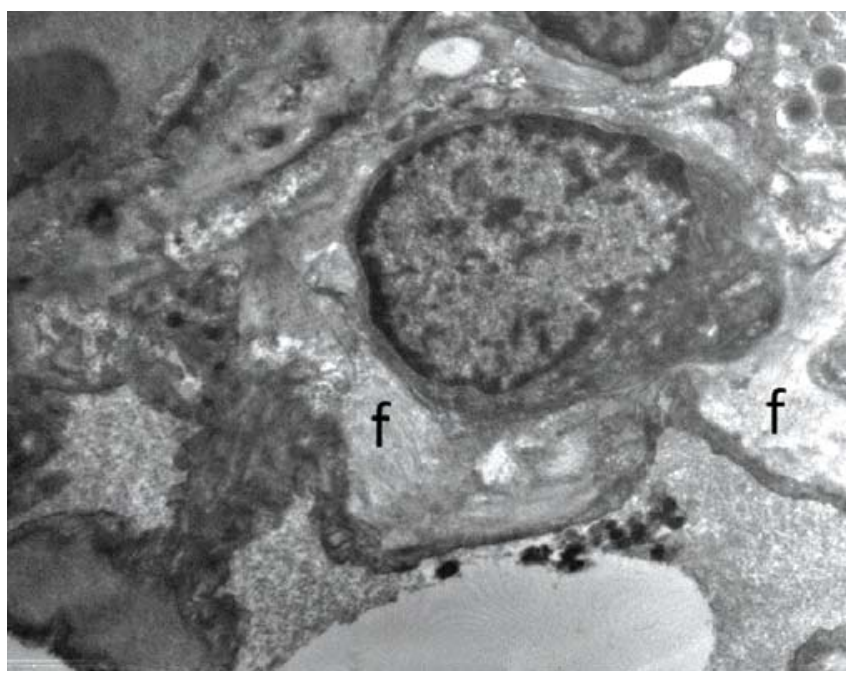

Figure 2. Transmission electron micrograph showing pulmonary fibrosis $(f)$ in hypoxia. (Original magnification $\times 5000$ ) (from the collection of author). 
teoglycans of the extracellular matrix and heparansulphate proteoglycans of the basement membrane were detected in acute hypoxia. There was a decrease in tissue elastance and an increase in the permeability of the endothelial barriers and epithelial barriers. In the study; when the overall proteoglycans fragmentation overcame to a critical threshold, developing of a severe lung edema was observed, in acute hypoxia.

In the adult mice; Reinke et al. ${ }^{[18]}$ examined the effects of chronic intermittent hypoxia on lung growth. The morphometry of the lungs, mean airspace chord lengths of lungs and alveolar surface areas of lungs were determined. In the study; it was shown that intermittent hypoxia increased the lung volumes and alveolar surface areas in the experimental model. Secondly; intermittent hypoxia induced a $60 \%$ increase in cellular proliferation, however; the number of proliferating type II alveolocytes were tripled. The authors could not be able to detect any increase in apoptosis. Other important findings of the study were upregulation of pathways for cellular movement, cellular growth and development in chronic intermittent hypoxia. Key developmental genes, vascular endothelial growth factor A and platelet-derived growth factor B were also upregulated by chronic intermittent hypoxia in adult mice. Zhang et al. ${ }^{[19]}$ studied the effects of hypoxia on the histological structure of rats' lungs. Their results demonstrated that mild edema and inflammatory cell infiltration appeared in the lung tissue at the third day of hypoxia. As the treated time extended; a gradually aggravated inflammation was observed in the rats. In the rats, due to the increase of the exposure time to normobaric hypoxia; interalveolar septa became thicker and thicker. By using in vivo high-resolution computed tomography measurements; Llapur et al ${ }^{[20]}$ examined the effects of chronic hypoxia on lung growth. In the study; a number of 19 healthy adults living at 2.000 meters above sea level and a number of 23 healthy adults living in 400 meters above sea level underwent high-resolution computed tomography. In between the two groups; differences in the total lung volume, air volume and lungs' tissue volume were compared. Additionally; the mean lung densities of the two groups, airway lumens and wall areas in anatomically matched airways were compared in between the two groups. The authors detected no significant differences for age, sex, weight or height in between the two groups. Their results showed that the adult lung did not increase its lung volume later in life by expansion of an existing number of alveoli.

Rivolta et al. ${ }^{[21]}$ developed a hypoxia model by applying $10 \%$ of oxygen to Wistar male rats for 30 days and compared the results with the control animals. The researchers measured: pulmonary interstitial pressure, cardiac and haemodynamic parameters by echocardiography. Additionally; they examined the morphometry of lungs in the lung specimens obtained after fixation in situ. After the exposure of hypoxia to rats; mean \pm standard deviation of pulmonary interstitial pressure was increased, air/tissue volume ratio was decreased and capillary vascularity index in the air-blood barrier was increased in hypoxic rats, when compared with the control group. In normal regions of the lung tissue; the density of pre-capillary vessels was increased and their thickness/internal diameter ratios were decreased. In the oedematous regions of the lungs; opposite results were obtained. In chronic hypoxia group of animals; the pulmonary artery pressure was increased, when compared to controls. In oedematous regions of lung tissue, the decrease in capillary vascularity was found to be correlated with the remarkable increase in interstitial pressure. Additionally; the morphometry of pre-capillary vessels suggested an increase in vascular resistance, in chronic hypoxia. In regions of normal lung tissue; the opposite findings were detected. In hypoxic state; an acute inflammatory reaction was observed in some studies found in the literature. The authors of these studies explained this condition by the activation of bone marrow and immigration of inflammatory precursor cells. It was measured by the increase of pulmonary granulocytes and megakaryocytes. ${ }^{[22-25]}$ In humans; the lack of oxygen resulted in a tremendous increase of perivascular mast cells, being rapidly recruited in the lungs within a few minutes. ${ }^{[26]}$ In the experimental study of Orth et al. ${ }^{[27]}$ performed on rats; the duration of hypoxia necessary for mast cell degranulation took only five minutes. Schmiedl et al. ${ }^{[9]}$ examined the lung parenchyma of 14-day old mice with light microscopy and electron microscopy. In the study; well-developed formed septa, normal alveoli and normal alveolar ducts were observed in the lungs of control group. Prenatal hypoxia induced lungs of mice showed lung parenchyma without clearly visible alterations, when compared to control animals. Postnatal hyperoxia induced lungs of mice indicated more expanded airspaces with fewer septa than the control group. In the microscopic examination; the lungs exposed to prenatal hypoxia and postnatal hyperoxia displayed enlarged parenchymal airspaces surrounded by thick septa. Hypoxia and hypoxia signalling have many negative effects on the lung tissue. Fibroblast proliferation, inflammatory cell infiltration and interstitial thickening are the main pathological findings. These pathological findings are combined with alveolar ventilation defects in order to increase the oxygen consumption and limit the oxygen supply to the injured lung tissues. As a result; 
local tissue hypoxia is observed in the lungs. In the lung tissues of patients having idiopathic pulmonary fibrosis or cystic fibrosis, and in the experimental models developed idiopathic pulmonary fibrosis or cystic fibrosis in mice; increased stabilisation of hypoxia-inducible transcription factor $(\mathrm{HIF}-1 \alpha)$ had been detected. ${ }^{[2,28,29]}$ Following exposure to hypoxia; a proliferation was observed in the fibroblasts and these fibroblasts had the highest quantity of excreted collagen; mainly the type-I collagen. ${ }^{[30]}$ As a result of all these studies; it can be concluded that hypoxia is involved in the development of pulmonary fibrosis (Figure 2). In an experimental study performed on rats; Ge et al. ${ }^{[31]}$ investigated the pathological changes on upper and lower respiratory tract in chronic intermittent hypoxia. In conclusion; chronic intermittent hypoxia formed irreversible changes both at upper and lower respiratory tracts in the rats. The thickness of the lamina propria of soft palate was significantly increased in hypoxia group. Additionally, in rats; pulmonary interval thickening was detected in the lungs.

\section{Hypoxia and Molecular Studies}

In hypoxic human lung fibroblast cell cultures, Shan-Shan Liu et al ${ }^{[32]}$ examined the effects and underlying mechanisms of angiotensin II on collagen synthesis. After hypoxic treatment; angiotensinogen, angiotensin converting enzyme, angiotensin II type 1 receptor and angiotensin II type 2 receptor expression levels in human lung fibroblast cell cultures were analysed by using real-time polymerase chain reaction. Secondly; collagen type I, angiotensin II type 1 receptor and nuclear factor kappaB $(\kappa \mathrm{B})$ protein expression levels were detected by using Western blot analysis. In addition to all these analyses; nuclear factor $\kappa \mathrm{B}$ nuclear translocation was measured by using immunofluorescence localization analysis. Angiotensin II levels in human lung fibroblast cell cultures were measured by using enzyme-linked immunosorbent assay (ELISA) method. The authors found that hypoxia increased collagen type I mRNA and protein expression levels in human lung fibroblast cells. According to the results of the study; it has been shown that these effects could be inhibited by an angiotensin II type 1 receptor or angiotensin II type 2 receptor inhibitor. After exposure to hypoxia; the levels of nuclear factor $\kappa \mathrm{B}$, renin-angiotensin system components and angiotensin II production in human lung fibroblast cell cultures were significantly increased. Tsao and $\mathrm{Wei}^{[3]}$ developed a prenatal hypoxia model in mice experimentally. In these animals; the authors examined the role of vascular endothelial growth factor (VEGF) and VEGF receptors in fetal lung development and maturation. In the study; prenatal hypoxia resulted in fetal mice body weight gain impairment and the authors observed delayed fetal pulmonary aeration and maturation in mice. Pulmonary surfactant proteins SP-A, SP-B, SP-C, and SP-D mRNA were all decreased in the prenatal hypoxia group in mice. In addition, the authors demonstrated that prenatal hypoxia inhibited the developmental increase of pulmonary hypoxia-inducible factors HIF-1 $\alpha$ and HIF$2 \alpha$ expression and resulted in decreasing VEGF and its receptors (Flt-1 and Flk-1) at the mRNA expression level. Secondly; the VEGF protein levels in fetal lungs were also found to be decreased.

Primary murine alveolar epithelial cells were exposed to $1 \%$ of oxygen for 24 hours in order to develop a hypoxia model in wild-type mice. In the study; a significant suppression of key innate immune molecules was observed. These key innate molecules included granulocytemacrophage colony stimulating factor, CCL2, and IL-6. Any toxicity finding was not observed in the experimental model, however; it induced stabilization of hypoxia inducible factor $1 \alpha$ protein and shift to glycolytic metabolism. The researchers found that hypoxia greatly decreased the rate of granulocyte-macrophage colony stimulating factor transcription. In another experimental model; the mice were exposed to hypoxia in vivo by the application of $12 \%$ oxygen for two days. In this model; the lung granulocyte-macrophage colony stimulating factor protein expression was reduced and in vivo phagocytosis of fluorescent beads by alveolar macrophages was suppressed. In conclusion of all these studies; it can be suggested that in critically ill patients; local hypoxia may contribute to the susceptibility of poorly ventilated lung units to infection through complementary effects on several pathways reducing alveolar epithelial cell expression of granulocytemacrophage colony stimulating factor and other key innate immune molecules. ${ }^{[34]}$ Many studies found in the literature revealed micro-RNAs to be involved in modulation of hypoxia-induced pulmonary hypertension. ${ }^{[35-37]}$ Blissenbach et al. ${ }^{[38]}$ examined the plasma micro-RNA levels in order to make a correlation with hypoxia induced pulmonary hypertension. In the study; plasma microRNAs were evaluated in a model of hypoxia-related pulmonary hypertension in humans exposed to extreme altitude. Plasma levels of micro-RNA -17, -21 , and -190 were measured by real-time quantitative PCR method. The obtained results were correlated with systolic pulmonary artery pressure, which was assessed by echocardiography. In the study; a significant altitude-dependent increase in circulating micro-RNAs expression was detected.

Four hours of exposure to a simulated altitude of 31,000 feet; Martinez-Romero et al. ${ }^{[3]]}$ studied compara- 
tively the effects of pharmacological inhibitors of nitric oxide production or Poly (ADP-ribose) polymerase activity in the response of the mouse cerebral cortex. The researchers analysed nitric oxide and reactive oxygen species production, the expression of nitric oxide synthase isoforms, Poly (ADP-ribose) polymerase-1 activity, hypoxia-inducible factor- 1 alpha expression and hypoxiainducible factor- 1 transcriptional activity, the protein level of the factor inhibiting hypoxia inducible factor, and at last beclin-1 and fractin expression, as markers of cellular damage. According to their results; the reduction of nitric oxide level did not affect reactive oxygen species production. However; significantly dampened the post hypoxic increase in neuronal nitric oxide synthase and inducible nitric oxide synthase expression without altering endothelial nitric oxide synthase protein level; prevented Poly (ADP-ribose) polymerase activation; decreased hypoxiainducible factor-1 alpha response to hypoxia; achieved a higher long-term hypoxia-inducible factor-1 transcriptional activity by reducing factor inhibiting hypoxiainducible factor expression and reduced hypoxic damage. Their results provided an important data about the molecular mechanisms underlying the beneficial effects of controlling nitric oxide production under hypobaric hypoxic conditions. Singh et al. ${ }^{[40]}$ examined the roles of activator protein-1 (AP-1) transcription factor; mitogen activated protein kinases (MAPKs) which are the c-Jun $\mathrm{N}$-terminal kinase (JNK), extracellular-signal regulated kinase (ERK) and $\mathrm{p} 38$ in the hypobaric hypoxia induced changes in lung tissue. In rats; immediate response to hypobaric hypoxia induced c-Jun:c-Fos subunits of AP-1; responsible for proliferation that might cause inhomogeneous vasoconstriction leading to vascular leakage and inflammation at increased duration of hypobaric hypoxia exposure. Secondly; hypoxia resulted in significant increase in reactive oxygen species (ROS), vascular endothelial growth factor (VEGF) and decreased nitric oxide (NO) in hypobaric hypoxia conditions. These act as signaling molecules for activation of MAPK and also contribute in development of vascular leakage which was an indicator of pulmonary edema.

In rats submitted to acute hypobaric hypoxia; Rus et al ${ }^{[41]}$ measured endothelial and inducible nitric oxide synthase (eNOS, iNOS) mRNA and protein expression, location, and in situ Nitric Oxide Synthase activity as well as nitrated protein expression and location. In addition, nitric oxide levels were indirectly quantified (NOx) as well as the apoptosis level. The researchers detected an increase in eNOS mRNA, protein activity and eNOS positive immunostaining at $0 \mathrm{~h}$ posthypoxia which coincided with raised NOx levels. However; the iNOS, nitrated pro- tein expression and apoptosis level augmented during the final reoxygenation times. The authors concluded that eNOS was responsible of the immediate response, producing nitric oxide, which caused vasodilation and bronchodilation in lung tissue. Secondly; iNOS was related to the second late response, which seemed to be involved in some of the deleterious consequences that hypoxia induced in the lung. In another study; by using a nitric oxide synthase (NOS) inhibitor, Rus et al. ${ }^{[42]}$ searched to clarify the current controversy concerning the double protective/toxic role of endogenous nitric oxide under hypox$\mathrm{ia} /$ reoxygenation situations. The study was conducted in rats submitted to hypoxia/reoxygenation with or without prior treatment using the nonselective NOS inhibitor LNAME. Their results showed that after L-NAME administration, in situ NOS activity was almost completely eliminated and consequently, NOx levels fell. Lipid peroxidation and the percentage of apoptotic cells rose at the earliest reoxygenation time, but decreased in the later periods, in the study.

\section{Hypoxia and Tumors}

Hypoxia occurs frequently in solid tumors and this condition is a poor prognostic factor for the outcome of the patients. Hypoxia promotes tumor cell proliferation, invasion of tumor cells, angiogenesis, resistance to therapeutic agents and occurrence of metastasis. In a growing tumor; two forms of hypoxia can be observed: Chronic hypoxia caused by abnormal tumor vasculature and intermittent hypoxia caused by transient perfusion facilitated by tumor-supplying blood vessels. In the presence of intermittent hypoxia; many genetic, molecular, biochemical, and cellular changes are detected. All of these changes facilitate the survival of tumor cells and their colonization. Depending upon to all these results; metastatic growth rate of the cancer cells increases. ${ }^{[43]}$

In order to reveal the possible mechanisms of brain metastases of lung cancers; Wei et al. ${ }^{[4]}$ studied the relationships in between hypoxia and hypoxia inducible factor- $1 \alpha(\mathrm{HIF}-1 \alpha)$ in lung cancer cells. The hypoxia model of A549 lung cancer cells was established in the study. After the hypoxia culture of A549 cells for 0.5, 2, 4, 8, 12 and 24 hours; the concentration of HIF- $1 \alpha$ in A549 lung cancer cell culture medium were determined by ELISA test. In conclusion; hypoxia induced the increase of HIF$1 \alpha$ in lung cancer cells. Due to the increase of HIF-1 $\alpha$ in A549 lung cancer cells; a decrease was detected in Claudin-5 expression. These changes increased bloodbrain barrier permeability which lead to the metastases of lung cancer cells into the brain tissue. 


\section{Conclusion}

Clinical manifestations of acute, intermittent or chronic hypoxia have negative effects on lung maturation during the embryological period which has been shown by many experimental models designed on animal studies. Hypoxia also affects the morphometry, anatomy and microscopy of lung tissue in the adults. Chronic hypoxia accentuates lung growth by increasing the lung parenchyma. However; the airways in the lung tissue were found to be normal in hypoxic cases. Histology of lung tissue shows microscopic changes due to hypoxia. Mild edema and inflammatory cell infiltration appear in the lung tissue in experimental studies performed on animal models. In cases of hypoxia; the lung parenchyma shows destructive exudative changes in the early phase; in the later phases repair and proliferative processes are dominant. Decrease of capillary volume, suppression of elastin repair in lung fibroblasts and pulmonary fibrosis are other clinically important findings of hypoxia. Due to hypoxia; the receptors responsible from fetal lung development are also affected. In molecular studies; hypoxia induced pulmonary hypertension, suppression of many immune molecules, major changes at the levels of various enzymes and growth factors were detected in the researches. Additionally; hypoxia causes to an increase in the amount of lung cancer cells and therefore; induces the metastases of lung cancer cells to brain tissues.

Exposure to hypoxia during embryological period must be avoided for normal lung maturation in the fetus. In the adult; if morphometric, anatomic or microscopic changes are observed in the lung tissue; exposure to hypoxia must also be remembered in differential diagnosis. If pulmonary hypertension, suppression of immune molecules, changes in the levels of enzymes or growth factors are detected in molecular studies of lung tissue; the possibility of the exposure to hypoxia must also be remembered. Additionally, in cases of lung cancers, in order to control the over increase of cancer cells or spread of metastases; a great care should be taken to keep patients away from hypoxic environment.

\section{Conflict of Interest}

The author declares that he has no conflict of interest.

\section{References}

1. Saladin KS. Anatomy and physiology. The unity of form and function. 8th ed. New York (NY): Mc Graw Hill Education; 2018. 878 p.

2. Tuder RM, Yun JH, Bhunia A, Fijalkowska I. Hypoxia and chronic lung disease. J Mol Med 2007;85:1317-24.

3. Schwartz JE, Kovach A, Meyer J, McConnell C, Iwamoto HS. Brief, intermittent hypoxia restricts fetal growth in Sprague-Dawley rats. Biol Neonate 1998;73:313-19.
4. Van Tuyl M, Liu J, Wang J, Kuliszewski M, Tibboel D, Post M. Role of oxygen and vascular development in epithelial branching morphogenesis of the developing mouse lung. Am J Physiol Lung Cell Mol Physiol 2005;288:L167-78.

5. Tufro-McReddie A, Norwood VF, Aylor KW, Botkin SJ, Carey RM, Gomez RA. Oxygen regulates vascular endothelial growth factor-mediated vasculogenesis and tubulogenesis. Dev Biol 1997;2: 139-49.

6. Maltepe E, Simon MC. Oxygen, genes, and development: an analysis of the role of hypoxic gene regulation during murine vascular development. J Mol Med (Berl) 1998;6:391-401.

7. Groenman FA, Rutter M, Wang J, Caniggia I, Tibboel D, Post M. Effect of chemical stabilizers of hypoxia-inducible factors on early lung development. Am J Physiol Lung Cell Mol Physiol 2007;3: L557-67.

8. Zhang H, Burggren WW. Hypoxic level and duration differentially affect embryonic organ system development of the chicken (Gallus gallus). Poult Sci 2012;91:3191-201.

9. Schmiedl A, Roolfs T, Tutdibi E, Gortner L, Monz D. Influence of prenatal hypoxia and postnatal hyperoxia on morphologic lung maturation in mice. PLoS One 2017;12:e0175804.

10. Davies P, Maddalo F, Reid L. Effects of chronic hypoxia on structure and reactivity of rat lung microvessels. J Appl Physiol 1985;58: 795-801.

11. Sekhon HS, Thurlbeck WM. Lung morphometric changes after exposure to hypobaria and/or hypoxia and undernutrition. Respir Physiol 1996;106:99-107.

12. Sulkowska M. Morphological studies of the lungs in chronic hypobaric hypoxia. Pol J Pathol 1997;48:225-34.

13. Clough AV, Haworth ST, Ma W, Dawson CA. Effects of hypoxia on pulmonary microvascular volume. Am J Physiol Heart Circ Physiol 2000;279:H1274-82.

14. Gade J, Qvortrup K, Andersen CB, Thorsen S, Svendsen UG, Olsen PS. Bronchial arterial devascularization. An experimental study in pigs. Scand Cardiovasc J 2001;35:212-20.

15. Gade J, Greisen G, Larsen IK, Bibby BM, Olsen PS. Tissue hypoxaemia causes oedema, inflammation and fibrosis in porcine bronchial transection. Scand Cardiovasc J 2012;46:286-94.

16. Berk JL, Hatch CA, Morris SM, Stone PJ, Goldstein RH. Hypoxia suppresses elastin repair by rat lung fibroblasts. Am J Physiol Lung Cell Mol Physiol 2005;289:L931-6.

17. Miserocchi G. Lung interstitial pressure and structure in acute hypoxia. Adv Exp Med Biol 2007;618:141-57.

18. Reinke C, Bevans-Fonti S, Grigoryev DN, Drager LF, Myers AC, Wise RA, Schwartz AR, Mitzner W, Polotsky VY. Chronic intermittent hypoxia induces lung growth in adult mice. Am J Physiol Lung Cell Mol Physiol 2011;300:L266-73.

19. Zhang X, Xie M, Gao Y, Wei HH, Zheng JQ. Study on the effect and mechanism of hypoxia on the histological structure of rat's lung [Article in Chinese]. Sichuan Da Xue Xue Bao Yi Xue Ban 2012;43: $1-5$.

20. Llapur CJ, Martínez MR, Grassino PT, Stok A, Altieri HH, Bonilla F, Caram MM, Krowchuk NM, Kirby M, Coxson HO, Tepper RS. Chronic hypoxia accentuates dysanaptic lung growth. Am J Respir Crit Care Med 2016;194:327-32.

21. Rivolta I, Lucchini V, Rocchetti M, Kolar F, Palazzo F, Zaza A, Miserocchi G. Interstitial pressure and lung oedema in chronic hypoxia. Eur Respir J 2011;37:943-9. 
22. Brinkmann B. Vital reactions of the pulmonary circulation in fatal strangulation. Z Rechtsmed 1978;81:133-46.

23. Brinkmann B, Püschel K. Histomorphological alterations of lung after strangulation. A comparative experimental study. Z Rechtsmed 1981;86:175-94.

24. Du Chesne A, Cecchi-Mureani R, Püschel K, Brinkmann B. Macrophage subtype patterns in protracted asphyxiation. Int J Legal Med 1996;109:163-6.

25. Strunk T, Hamacher D, Schulz R, Brinkmann B. Reaction patterns of pulmonary macrophages in protracted asphyxiation. Int J Legal Med 2010;124:559-68.

26. Muciaccia B, Sestili C, De Grossi S, Vestri A, Cipolloni L, Cecchi R. Are mast cells implicated in asphyxia? Int J Legal Med 2016;130: 153-61.

27. Orth T, Allen JA, Wood JG, Gonzalez NC. Plasma from conscious hypoxic rats stimulates leukocyte-endothelial interactions in normoxic cremaster venules. J Appl Physiol (1985) 2005;99:290-7.

28. Urquhart DS, Montgomery H, Jaffé A. Assessment of hypoxia in children with cystic fibrosis. Arch Dis Child 2005;90:1138-43.

29. Tzouvelekis A, Harokopos V, Paparountas T, Oikonomou N, Chatziioannou A, Vilaras G, Tsiambas E, Karameris A, Bouros D, Aidinis V. Comparative expression profiling in pulmonary fibrosis suggests a role of hypoxia-inducible factor-1alpha in disease pathogenesis. Am J Respir Crit Care Med 2007;176:1108-19.

30. Ahmedat A, Warnken M, Seemann W, Mohr K, Kostenis E, Juergens U, Racké K. Profibrotic processes in human lung fibroblasts are driven by an autocrine/paracrine endothelinergic system. $\mathrm{Br}$ J Pharmacol 2013;168:471-87.

31. Ge L, Ming T, Hou J, Yan J, Zhao L, Gong N, Jiang J, Wang F. Pathological changes of upper and lower respiratory tissue in rats with chronic intermittent hypoxia [Article in Chinese]. Zhonghua Er Bi Yan Hou Tou Jing Wai Ke Za Zhi (Chinese Journal of Otorhinolaryngology Head and Neck Surgery) 2015;50:939-43.

32. Liu SS, Wang HY, Tang JM, Zhou XM. Hypoxia-induced collagen synthesis of human lung fibroblasts by activating the angiotensin system. Int J Mol Sci 2013;14:24029-45.

33. Tsao PN, Wei SC. Prenatal hypoxia downregulates the expression of pulmonary vascular endothelial growth factor and its receptors in fetal mice. Neonatology 2013;103:300-7.
34. Sturrock A, Woller D, Freeman A, Sanders K, Paine R. Consequences of hypoxia for the pulmonary alveolar epithelial cell innate immune response. J Immunol 2018;201:3411-20.

35. Li Q, Zhou X, Zhou X. Downregulation of miR 98 contributes to hypoxic pulmonary hypertension by targeting ALK1. Mol Med Rep 2019;20:2167-76.

36. Liu T, Zou XZ, Huang N, Ge XY, Yao MZ, Liu H, Zhang Z, Hu CP. miR-27a promotes endothelial-mesenchymal transition in hypoxia-induced pulmonary arterial hypertension by suppressing BMP signaling. Life Sci 2019;227:64-73.

37. Liu A, Liu Y, Li B, Yang M, Liu Y, Su J. Role of miR-223-3p in pulmonary arterial hypertension via targeting ITGB3 in the ECM pathway. Cell Prolif 2019;52:e12550.

38. Blissenbach B, Nakas CT, Krönke M, Geiser T, Merz TM, Pichler Hefti J. Hypoxia-induced changes in plasma micro-RNAs correlate with pulmonary artery pressure at high altitude. Am J Physiol Lung Cell Mol Physiol 2018;314:L157-64.

39. Martínez-Romero R, Cañuelo A, Siles E, Oliver FJ, Martínez-Lara E. Nitric oxide modulates hypoxia-inducible factor- 1 and poly (ADP-ribose) polymerase-1 cross talk in response to hypobaric hypoxia. J Appl Physiol 2012;112:816-23.

40. Singh M, Yadav S, Kumar M, Saxena S, Saraswat D, Bansal A, Singh $\mathrm{SB}$. The MAPK-activator protein-1 signaling regulates changes in lung tissue of rat exposed to hypobaric hypoxia. J Cell Physiol 2018;233:6851-65.

41. Rus A, Peinado MA, Castro L, Del Moral ML. Lung eNOS and iNOS are reoxygenation time-dependent upregulated after acute hypoxia. Anat Rec 2010;293:1089-98.

42. Rus A, Molina F, Peinado MA, Del Moral ML. Endogenous nitric oxide can act as beneficial or deleterious in the hypoxic lung depending on the reoxygenation time. Anat Rec 2010;293:2193201.

43. Chen A, Sceneay J, Gödde N, Kinwel T, Ham S, Thompson EW, Humbert PO, Möller A. Intermittent hypoxia induces a metastatic phenotype in breast cancer. Oncogene 2018;37:4214-25.

44. Wei DF, Tang MK, Liu Y, Zhang CY, Qin LJ. Effect of hypoxia inducible Factor-1 alpha on brain metastasis from lung cancer and its mechanism [Article in Chinese]. Sichuan Da Xue Xue Bao Yi Xue Ban 2019;50:188-92.
ORCID ID: M. F. Sargon 0000-0001-6360-6008

\author{
Correspondence to: Mustafa F. Sargon, MD, PhD. \\ Department of Anatomy, Faculty of Medicine, \\ Lokman Hekim University, Ankara, Turkey \\ Phone: +90 5053658196 \\ Conflict of interest statement: No conflicts declared.
}

This is an open access article distributed under the terms of the Creative Commons Attribution-NonCommercial-NoDerivs 4.0 Unported (CC BY-NCND4.0) Licence (http://creativecommons.org/licenses/by-nc-nd/4.0/) which permits unrestricted noncommercial use, distribution, and reproduction in any medium, provided the original work is properly cited. How to cite this article: Sargon MF. Lungs and hypoxia: a review of the literature. Anatomy 2021;15(1):76-83. 